\title{
Effects of Intermittent Administration of Parathyroid Hormone (1-34) on Bone Differentiation in Stromal Precursor Antigen-1 Positive Human Periodontal Ligament Stem Cells
}

\author{
Xiaoxiao Wang, ${ }^{1}$ Yanlan Wang, ${ }^{1,2}$ Xubin Dai, ${ }^{1}$ Tianyu Chen, \\ Fanqiao Yang, ${ }^{1}$ Shuangye Dai, ${ }^{1}$ Qianmin Ou, ${ }^{1}$ Yan Wang, ${ }^{1}$ and Xuefeng Lin ${ }^{1}$ \\ ${ }^{1}$ Guanghua School of Stomatology, Sun Yat-sen University, Guangdong Provincial Key Laboratory of Stomatology, \\ Guangzhou 510080, China \\ ${ }^{2}$ School and Hospital of Stomatology, Wenzhou Medical University, Wenzhou 325035, China \\ Correspondence should be addressed to Yan Wang; wang93@mail.sysu.edu.cn and Xuefeng Lin; linxfeng@mail.sysu.edu.cn
}

Received 11 October 2015; Accepted 17 February 2016

Academic Editor: Armand Keating

Copyright (c) 2016 Xiaoxiao Wang et al. This is an open access article distributed under the Creative Commons Attribution License, which permits unrestricted use, distribution, and reproduction in any medium, provided the original work is properly cited.

\begin{abstract}
Periodontitis is the most common cause of tooth loss and bone destruction in adults worldwide. Human periodontal ligament stem cells (hPDLSCs) may represent promising new therapeutic biomaterials for tissue engineering applications. Stromal precursor antigen-1 (STRO-1) has been shown to have roles in adherence, proliferation, and multipotency. Parathyroid hormone (PTH) has been shown to enhance proliferation in osteoblasts. Therefore, in this study, we aimed to compare the functions of STRO-1(+) and STRO-1(-) hPDLSCs and to investigate the effects of PTH on the osteogenic capacity of STRO-1(+) hPDLSCs in order to evaluate their potential applications in the treatment of periodontitis. Our data showed that STRO-1(+) hPDLSCs expressed higher levels of the PTH-1 receptor (PTH1R) than STRO-1(-) hPDLSCs. In addition, intermittent PTH treatment enhanced the expression of PTH1R and osteogenesis-related genes in STRO-1(+) hPDLSCs. PTH-treated cells also exhibited increased alkaline phosphatase activity and mineralization ability. Therefore, STRO-1(+) hPDLSCs represented a more promising cell resource for biomaterials and tissue engineering applications. Intermittent PTH treatment improved the capacity for STRO-1(+) hPDLSCs to repair damaged tissue and ameliorate the symptoms of periodontitis.
\end{abstract}

\section{Introduction}

Periodontitis is the most common cause of irreversible destruction of periodontal tissue and tooth loss in adults worldwide. However, conventional periodontal therapy for the treatment of periodontal tissue damage does not induce the regeneration of periodontal supporting tissue $[1,2]$. Therefore, the development of regenerative therapies for the treatment of periodontal disease has become a major challenge [3].

Several studies have suggested that mesenchymal stem cells (MSCs) may represent promising therapies for the functional repair of defect and injury caused by periodontitis [4, 5]. Recently, dental tissue-derived stem cell therapy has been shown to be a promising new method for the regeneration of periodontal tissue [3]. Periodontal ligament stem cells (PDLSCs), a unique population of MSCs found in periodontal ligaments, are easy to obtain and can be induced into osteoblast-like cells and adipocytes in vitro [6]. In addition, PDLSCs are currently the most favorable candidates for the treatment of advanced periodontitis, showing superiority when compared with dental pulp stem cells (DPSCs) and periapical follicular stem cells (PAFSCs) [7]. Moreover, in a swine model, researchers transplanted human PDLSCs (hPDLSCs) into alveolar bone defect areas and found that periodontal tissue was eventually repaired and regenerated [4], supporting the potential of these cells in tissue regeneration.

Stromal precursor antigen-1 (STRO-1) is a widely used marker of MSCs [8-10]. STRO-1 was found to be related 
to adherence, proliferation, and multilineage differentiation potential $[10,11]$, indicating that STRO-1(+) cells may represent a unique subgroup of hPDLSCs with specific functions in the healing of periodontitis-associated bone damage.

Parathyroid hormone (PTH), a peptide hormone produced at the endoplasmic reticulum, regulates calcium and phosphorus metabolism and mineral homeostasis [12]. A recent study showed that $\mathrm{PTH}$ binds to the $\mathrm{PTH}$ receptor on MSCs, committing MSCs to the osteoblast lineage and promoting bone formation [13]. Intermittent administration of PTH (1-34) has been shown to increase bone-to-implant contact, induce new bone formation around the implants, and increase bone mineral density [14]. Furthermore, PTH (1-34) stimulates new bone formation, leading to enhanced periodontal healing [15].

In this study, we hypothesized that PTH would affect the osteogenic capacity of PDLSCs and show the potential for application as a cell source in periodontitis treatment. Therefore, we isolated and characterized STRO-1(+) hPDLSCs, compared the levels of PTH receptor in STRO$1(+)$ and STRO-1(-) PDLSCs, and evaluated the effects of PTH treatment on the differentiation capacity of STRO-1(+) PDLSCs.

\section{Materials and Methods}

2.1. Cell Culture and PTH Treatment. Healthy premolars were collected from 10 adults (15-20 years of age; five men and five women) for orthodontic purposes at the Department of Oral and Maxillofacial Surgery, the Affiliated Stomatological Hospital of Sun Yat-sen University. All participants provided informed consent for the collection and use of their tissues. The protocols were approved by the University Ethics Committee.

PDLSCs were isolated and cultured as previously reported [6]. Briefly, periodontal tissue was gently separated from the surface of the middle third of the root and then digested with $3 \mathrm{mg} / \mathrm{mL}$ collagenase type I and $4 \mathrm{mg} / \mathrm{mL}$ dispase (GibcoBRL, Gaithersburg, MD, USA) at $37^{\circ} \mathrm{C}$ for $1 \mathrm{~h}$. Colonyforming cells were collected and then cultured in alpha modified Eagle medium (Gibco-BRL) supplemented with 10\% fetal bovine serum (Gibco-BRL), $100 \mu \mathrm{g} / \mathrm{mL}$ streptomycin, $100 \mathrm{U} / \mathrm{mL}$ penicillin (Hyclone, Logan, UT, USA), $200 \mu \mathrm{M}$ L-ascorbic acid (Sigma-Aldrich, St. Louis, MO, USA), and $5 \mathrm{mM}$ L-glutamine (Gibco-BRL) at $37^{\circ} \mathrm{C}$ in an atmosphere containing $5 \% \mathrm{CO}_{2}$. The medium was changed every 3 days. Cells were used at passages 3-5. For intermittent PTH treatment, cells were treated with $10^{-12} \mathrm{M} \mathrm{PTH} \mathrm{(1-34)} \mathrm{(Sigma-}$ Aldrich) for $6 \mathrm{~h}$, followed by another $6 \mathrm{~h}$ treatment after $32 \mathrm{~h}$. Cells were harvested at various times after treatment with $10^{-12} \mathrm{M}$ PTH (1-34) for RNA and protein isolation.

2.2. Flow Cytometry. For flow cytometry, $5 \times 10^{5}$ cells were collected and washed with phosphate-buffered saline (PBS). Cells were then incubated with PE-labeled anti-CD90, antiCD105, anti-CD166, and anti-CD34 antibodies (1:10; Becton Dickinson Biosciences, San Jose, CA, USA) and with FITClabeled anti-CD146 antibodies (1: 10; Life Technologies Corp.,
Carlsbad, CA, USA) at room temperature for $1 \mathrm{~h}$ in the dark. Cells were incubated with STRO-1 IgM (1:10; Life Technologies Corp.) at room temperature for $1 \mathrm{~h}$ and then incubated with PE-conjugated anti-IgM (1:500; Life Technologies Corp.) for $30 \mathrm{~min}$ in the dark. After incubation, cells were washed three times with PBS and resuspended in $300 \mu \mathrm{L}$ PBS. Flow cytometry was carried out using a BD Accuri C6 (Becton Dickinson Biosciences). Data were analyzed using CF Low Plus Software (Becton Dickinson Biosciences).

2.3. Pluripotency of hPDLSCs. PDLSCs (passage 3) were seeded at a density of $1 \times 10^{5}$ cells/well in 12 -well plates. For osteogenic differentiation, after reaching 70-80\% confluence, cells were cultured in osteogenic-induction medium (Cyagen Biosciences Inc., Santa Clara, CA, USA) according to the manufacturer's protocol. Three weeks later, cultures were fixed with $4 \%$ paraformaldehyde for $15 \mathrm{~min}$ and stained with Alizarin red; quantification of mineralization was performed as previously described [16]. For adipogenic differentiation, cells were cultured in adipoinductive medium (Cyagen Biosciences Inc.) according to the manufacturer's protocol. Two weeks later, cells were fixed with $4 \%$ paraformaldehyde for 30 min and stained with Oil Red O.

2.4. Immunomagnetic Cell Sorting. hPDLSCs were collected at passage 3, and STRO-1(+) cells were isolated using Dynabeads rat anti-mouse IgM (Life Technologies) according to the manufacturer's instructions. Briefly, $1 \times 10^{7}$ cells were resuspended in $1 \mathrm{~mL}$ PBS with $0.1 \%$ bovine serum albumin, $2 \mathrm{mM}$ EDTA, and $25 \mu \mathrm{L}$ washed rat anti-mouse magnetic Dynabeads. Next, $10 \mu \mathrm{L}$ of anti-human STRO-1 IgM antibodies (Life Technologies) was added, and cells were incubated at $4^{\circ} \mathrm{C}$ for $1 \mathrm{~h}$. The tube was then placed in a magnet for $2 \mathrm{~min}$, and the bead-bound STRO-1(+) cells and bead-free STRO-1(-) cells were isolated.

2.5. Cell Immunofluorescence Staining. At passage 4, STRO$1(+)$ and STRO-1(-) hPDLSCs $\left(1 \times 10^{4}\right)$ were cultivated overnight on cover slips (NEST Biotech Co. Ltd., Shanghai, China). Cells were washed with PBS, fixed in $4 \%$ paraformaldehyde at room temperature for $10 \mathrm{~min}$, incubated with blocking solution containing $1 \%$ BSA for $30 \mathrm{~min}$, and then incubated with primary anti-STRO-1 IgM (1:100; Life Technologies) and anti-PTH1R IgG antibodies (1:50; Cambridge, UK) overnight at $4^{\circ} \mathrm{C}$. Cells were then incubated with Alexa Fluor 488-conjugated anti-mouse IgM or IgG (1:250; Life Technologies) for $45 \mathrm{~min}$ at room temperature. Cell nuclei were counterstained with DAPI (Life Technologies) and photographed with Zeiss Axio Observer Z1 (Carl Zeiss, Oberkochen, Germany).

2.6. Quantitative Real-Time Reverse Transcription Polymerase Chain Reaction (RT-qPCR). Total RNA was extracted at various times using TRIzol reagent (Life Technologies), and first-strand cDNA was synthesized using a reverse transcriptase M-MLV Kit (TaKaRa, Shiga, Japan). Gene expression was quantified by RT-qPCR using a SYBR Green kit (Roche, Basel, Switzerland) with gene-specific primers 
for the detection of $P T H 1 R$, runt-related transcription factor 2 (RUNX2), Sp7 (also known as Osterix), and GAPDH. The cycling parameters were as follows: $95^{\circ} \mathrm{C}$ for $10 \mathrm{~min}$, followed by 40 cycles at $95^{\circ} \mathrm{C}$ for $15 \mathrm{~s}, 60^{\circ} \mathrm{C}$ for $20 \mathrm{~s}$, and $72^{\circ} \mathrm{C}$ for $20 \mathrm{~s}$. The primers used in this study were as follows: PTH1R: forward, $5^{\prime}$-AGTGCGAAAAACGGCTCAAG$3^{\prime}$, and reverse, $5^{\prime}$-GATGCCTTATCTTTCCTGGGC-3'; RUNX2: forward, $5^{\prime}$-TGGTTACTGTCATGGCGGGTA- ${ }^{\prime}$, and reverse, $5^{\prime}$-TCTCAGATCGTTGAACCTTGCTA- $3^{\prime}$; SP7: forward, $5^{\prime}$-CCTCTGCGGGACTCAACAAC- $3^{\prime}$, and reverse, $5^{\prime}$-AGCCCATTAGTGCTTGTAAAGG-3'; and GAPDH: forward, $5^{\prime}$-AGGTCGGAGTCAACGGATTTG-3', and reverse, $5^{\prime}$-AGGCTGTTGTCATACTTCTCAT- ${ }^{\prime}$. The primer pair for human $P T H 1 R$ was designed using the Primer Premier 5.0 program based on the cDNA sequence data. The primers for $R U N X 2, S P 7$, and GAPDH were previously described by Sakaki-Yumoto et al. [17], Zhang et al. [18], and Nozell and Chen [19].

2.7. Western Blotting. Cell lysates containing $40 \mu \mathrm{g}$ total protein were loaded on sodium dodecyl sulfate polyacrylamide gels, separated by electrophoresis, and transferred to nitrocellulose membranes. Membranes were blocked with $5 \%$ nonfat dry milk for $2 \mathrm{~h}$ at room temperature and then incubated with anti-PTH1R (1:100; Boster, Wuhan, Hubei, China), anti-RUNX2 (1:100; Boster), anti-Sp7 (1:250; SigmaAldrich), and anti- $\beta$-actin (1:5000; Sigma-Aldrich) antibodies overnight at $4^{\circ} \mathrm{C}$. Membranes were washed, incubated with IRDye 680LT goat anti-mouse or goat anti-rabbit IgG (1:3000; LI-COR Biosciences, Lincoln, NE, USA) for $30 \mathrm{~min}$, and scanned using an Odyssey two-color infrared laser imaging system (LI-COR). Each experiment was performed in triplicate.

2.8. Alkaline Phosphatase (ALP) Activity. ALP activity was measured after 8 days of osteogenic differentiation. Cells were harvested with lysis buffer $(20 \mathrm{mM}$ Tris- $\mathrm{HCl}, 150 \mathrm{mM} \mathrm{MgCl}$, $1 \%$ Triton X-100, and $1 \mathrm{mM}$ phenylmethylsulfonyl fluoride). Total protein concentrations were determined using a Pierce BCA Protein Assay Kit (Life Technologies). Fifty microliters of supernatant was added to $50 \mu \mathrm{L} p$-nitrophenyl phosphate hexahydrate ( $1 \mathrm{~g} / \mathrm{L}$; Sigma-Aldrich) containing $1 \mathrm{mM} \mathrm{MgCl}_{2}$, and the mixture was incubated at $37^{\circ} \mathrm{C}$ for $30 \mathrm{~min}$. Absorption at $405 \mathrm{~nm}$ was measured in duplicate well with a microplate reader (BioTek Instruments, Inc., Winooski, VT, USA). ALP activity per total protein $(\mu \mathrm{g})$ represented the millimoles of $p$-nitrophenol released after a $30 \mathrm{~min}$ incubation at $37^{\circ} \mathrm{C}$.

\section{Results}

3.1. Characterization of hPDLSCs. hPDLSCs were isolated from the root surface of premolars and cultured. The colonyforming hPDLSCs displayed a uniform fibroblast-like morphology (Figure 1(a)). Culture-expanded hPDLSCs exhibited osteogenic and adipogenic differentiation potential, as shown by the formation of mineralized nodules and lipid-containing adipocytes (Figure $1(\mathrm{~b})$ ).
MSC markers on hPDLSCs were then measured by flow cytometry. The majority of the adherent cells expressed MSC markers, including CD90 (96.0\%), CD105 (98.0\%), CD166 (98.5\%), CD146 (26.4\%), and STRO-1 (8.76\%), but were negative for the hematopoietic cell marker CD34 (0.966\%; Figure 1(c)), suggesting that the isolated hPDLSCs were indeed stem cells of mesenchymal origin.

3.2. STRO-1(+) hPDLSCs Exhibited Stronger Osteogenic Ability. A recent study showed that STRO-1(+) cells exhibit higher adherence, proliferation, and multipotency [11]. Therefore, we next analyzed the characteristics of STRO-1(-) and STRO-1(+) hPDLSCs after isolation of both cell types. A distinct positive immunoreaction for STRO-1 was observed in STRO-1(+) hPDLSCs but not in STRO-1(-) hPDLSCs (Figure 2(a)).

We then conducted ALP activity and Alizarin red staining assays to compare the differentiation potential between STRO-1(+) and STRO-1(-) hPDLSCs. Our results showed that the ALP activity of STRO-1(+) hPDLSCs was significantly higher than that of STRO-1(-) hPDLSCs (Figure 2(b)). Alizarin red staining on day 21 revealed that the mineralized nodules of STRO-1 $(+)$ cells were larger (Figure 2(c)). These data were supported by the results of RT-qPCR analysis and western blotting (Figure 2(d)). Indeed, the expression of RUNX2, a typical osteogenesis-related gene, was higher in STRO-1(+) cells than in STRO-1(-) cells (Figure 2(d)). Thus, our data suggested that STRO-1(+) hPDLSCs exhibited higher differentiation potential.

3.3. PTH Treatment Enhanced the Osteogenesis of STRO-1(+) hPDLSCs. We next compared the expression levels of PTH1R in STRO-1(+) and STRO-1(-) hPDLSCs. Interestingly, the mRNA and protein levels of PTH1R in STRO-1(+) hPDLSCs were higher than those in STRO-1(-) hPDLSCs (Figure 3), indicating that STRO-1(+) cells may be more sensitive to PTH1R treatment, which could therefore affect the bone differentiation potential and suitability for tissue engineering.

To test this hypothesis, we treated the cells with PTH intermittently and detected changes in the expression of osteogenesis-related genes. After 8 days of PTH treatment and osteogenic induction, the expression levels of osteogenesis-related genes were quantified by RT-qPCR and western blotting. After induction, the expressions of PTH1R, RUNX2, and SP7 were upregulated by PTH treatment (Figure 4(a)). Moreover, intermittent PTH treatment elevated $S P 7$ expression in both induced and uninduced cells, and PTH increased the expression of PTH1R, creating a positive feedback loop (Figure 4(a)).

We then used ALP activity assays to determine whether PTH affected osteogenic differentiation. PTH-treated cells exhibited stronger osteogenic differentiation potential $(P<$ 0.01; Figure 4(b)). After 22 days of induction, the formation of mineralized nodules was observed and measured. Control cells formed small mineralized nodules in terms of both area and density. In contrast, in cells treated with osteogenic medium and PTH, the formation of mineralized nodules was significantly increased in terms of both area and intensity 


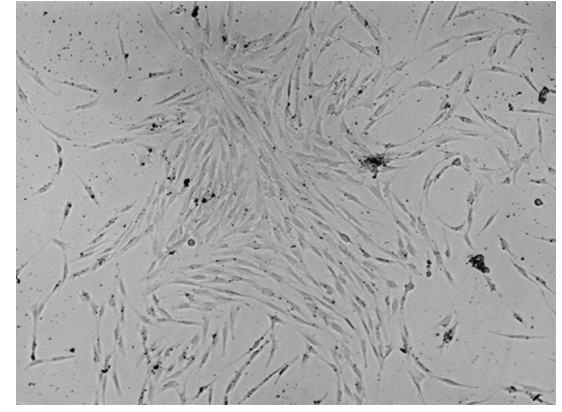

(a)
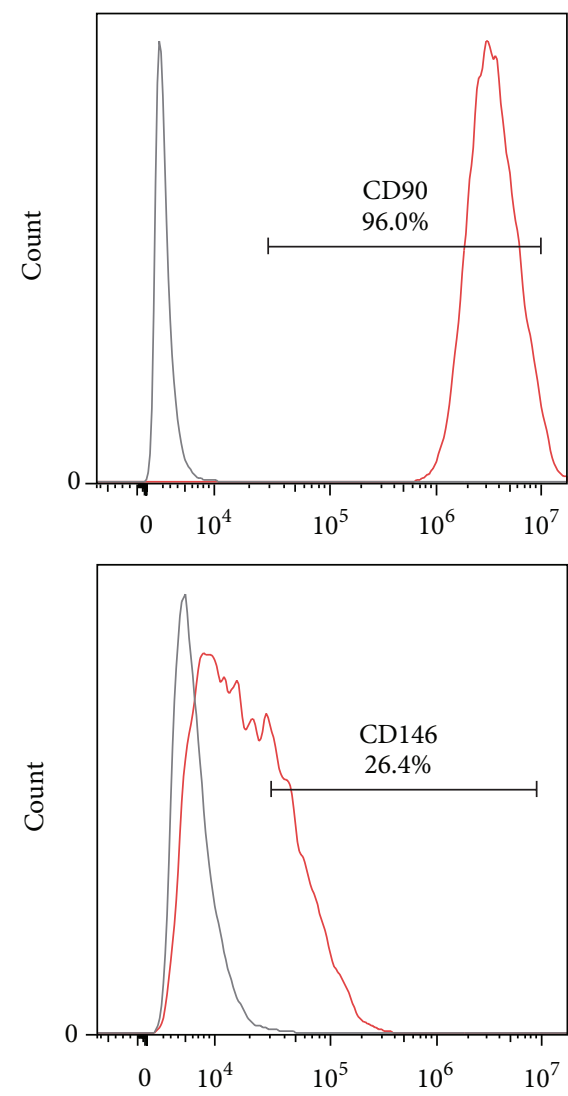
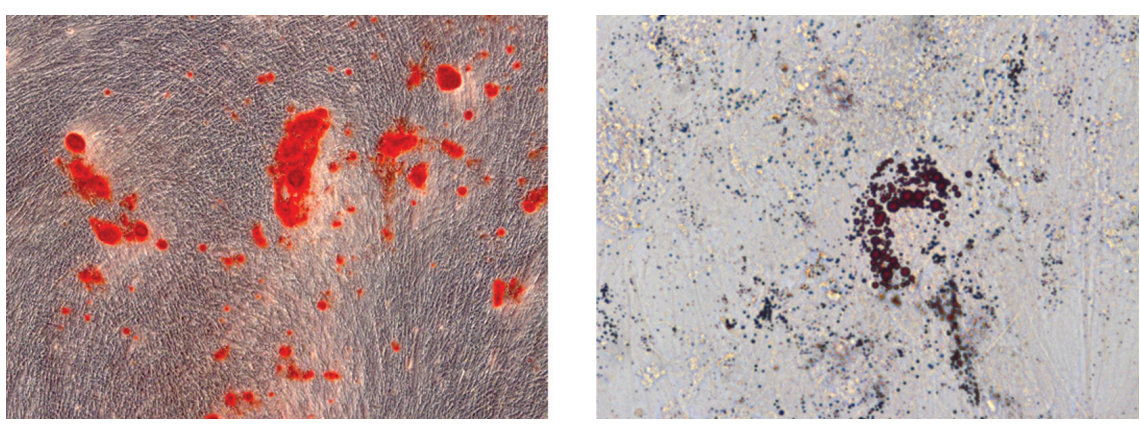

(b)
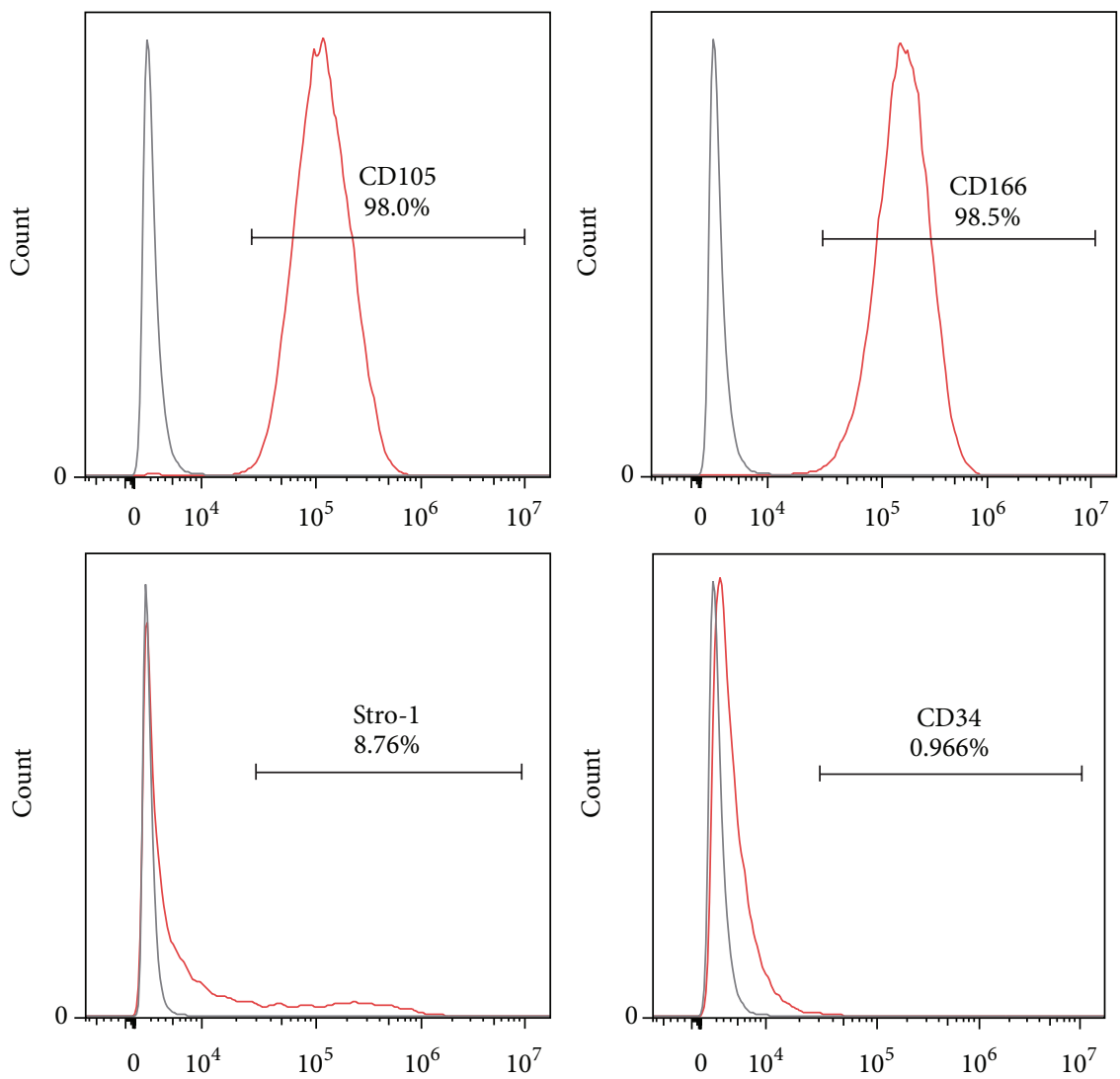

(c)

FIGURE 1: Characterization of hPDLSCs. (a) The morphology of colony-forming hPDLSCs (magnification: 50x). (b) Differentiation potential of hPDLSCs. Differentiated cells were stained with Alizarin red (left; magnification: 100x) or Oil Red O (right; magnification: 630x). (c) Immunophenotypic profiling was performed to detect CD90, CD105, CD166, CD146, Stro-1, and CD34.

(Figure 4(c)). Taken together, our data showed that intermittent treatment with PTH significantly enhanced osteogenesis in STRO-1(+) hPDLSCs.

\section{Discussion}

In this study, we aimed to determine whether PTH affected the osteogenic capacity of STRO-1(+) PDLSCs in order to analyze the potential applications of these cells in the treatment of periodontitis. Our results showed that PTH could stimulate osteogenesis in STRO-1(+) hPDLSCs, suggesting that this population of cells could be used in tissue regeneration to repair periodontitis-dependent bone damage.

We isolated PDLSCs from periodontal ligaments and evaluated their biological and immunological properties. Colony-forming cells expressed the surface markers CD90, CD105, and CD166 at high frequencies (95\%) but rarely expressed CD34 (less than 2\%). Moreover, the cells exhibited osteogenic and adipogenic differentiation potential. These results indicated that we had successfully isolated PDLSCs, 


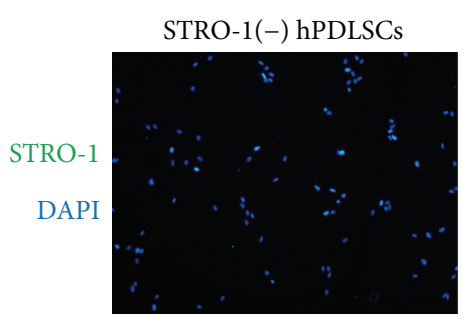

(a)

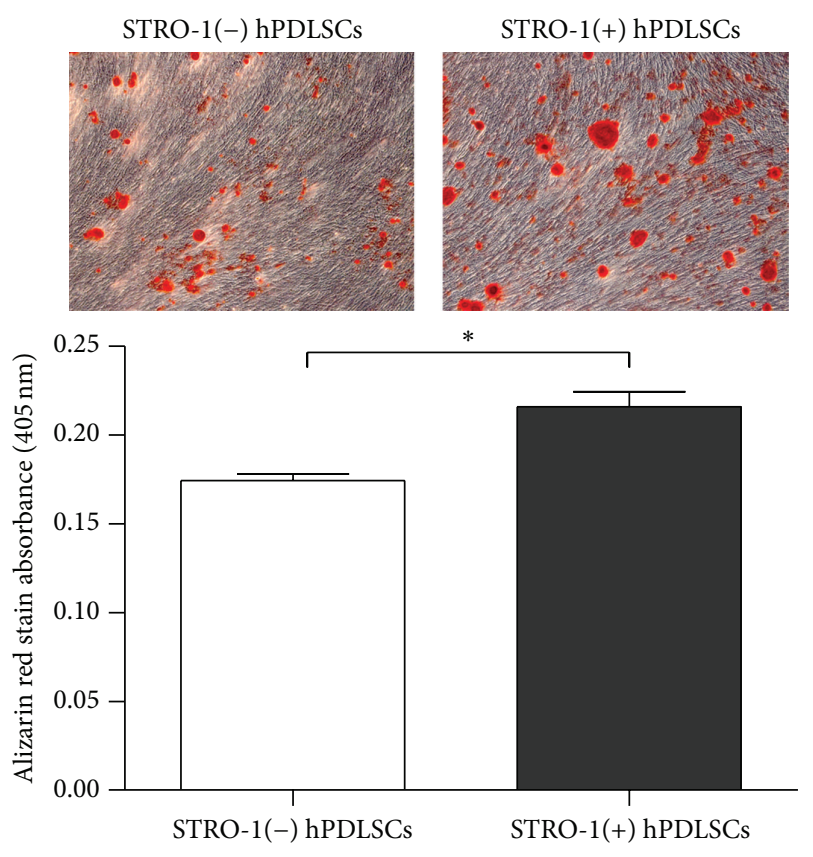

(c)

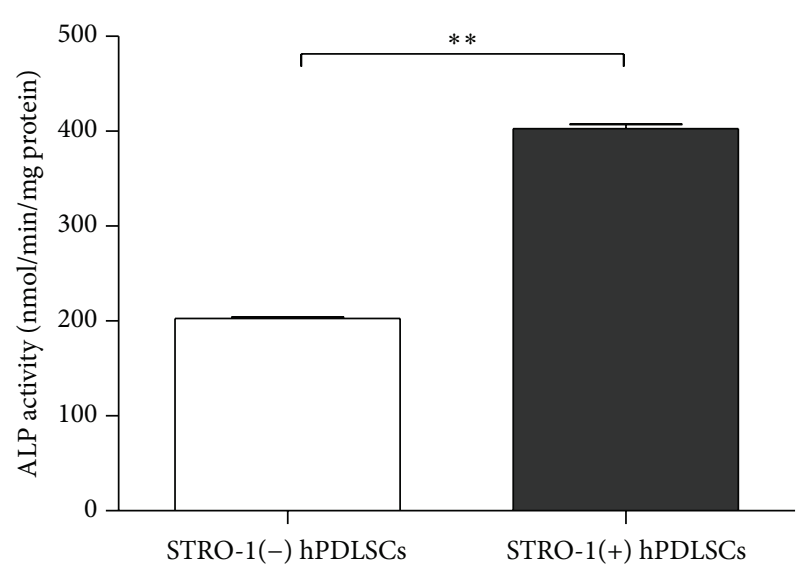

(b)

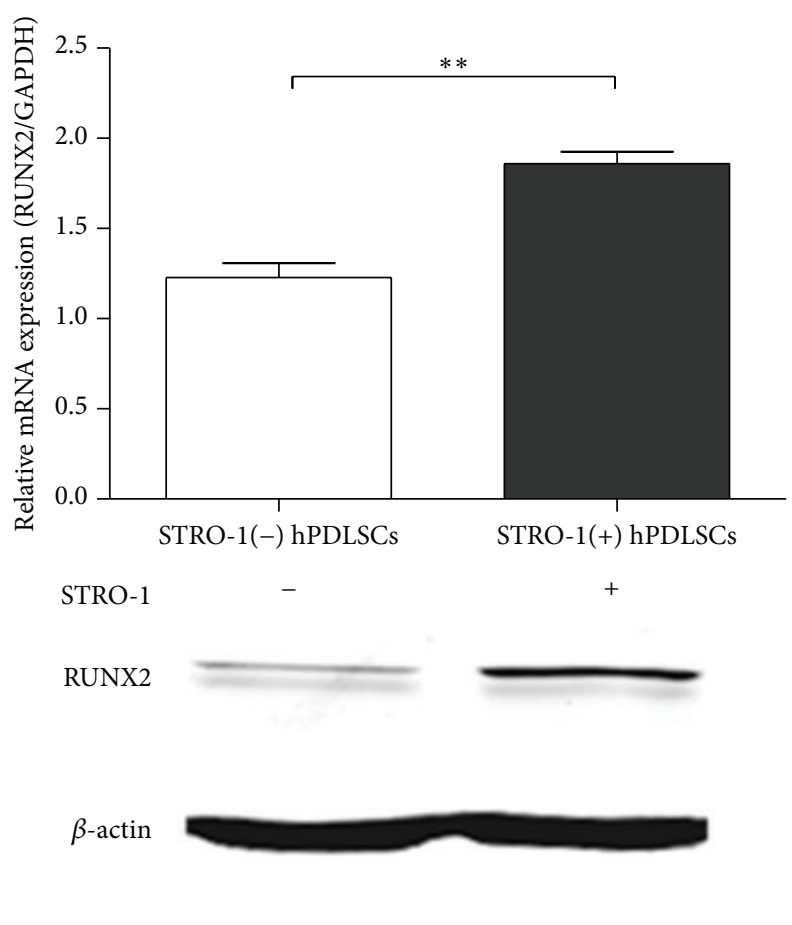

(d)

FIGURE 2: STRO-1(+) hPDLSCs exhibited strong osteogenic capacity. (a) Immunostaining for Stro-1 in isolated STRO-1(+) and STRO-1(-) hPDLSCs (magnification: 50x). The surface marker Stro-1 was stained with FITC and visualized using fluorescence microscopy. Nuclei were stained with DAPI (blue). (b) ALP activity in STRO-1(+) and STRO-1(-) hPDLSCs. (c) Osteogenic differentiated cells were stained with Alizarin red (magnification: 100x). (d) Real-time PCR and western blot analyses of Runx2 expression in STRO-1(+) and STRO-1(-) hPDLSCs. The expression of each target was normalized to that of GAPDH. Data are presented as the means \pm SDs of three independent experiments performed in duplicate. ${ }^{*} P<0.05,{ }^{* *} P<0.01$.

according to the criteria presented in the International Society for Cellular Therapy position statement $[6,20]$. With the capacity for self-renewal and differentiation into osteoblastlike cells, PDLSCs have become a promising source for regeneration of tissue in the treatment of bone defects $[4,21]$.

STRO-1(+) cells exhibited a more primitive phenotype $[22,23]$. We found that $8.76 \%$ of total hPDLSCs were STRO$1(+)$, consistent with previous studies (6.7-20\%) [24-26]. The purified STRO-1(+) cells exhibited higher differentiation potential, supporting the hypothesis that STRO-1(+) and STRO-1(-) cells represent different subgroups of cells with different functions in differentiation $[27,28]$. Similarly, in previous studies, the osteogenic ability of STRO-1(+) hPDLSCs was found to be significantly higher than that of unsorted cells $[29,30]$. In particular, we found that STRO1(+) hPDLSCs expressed higher levels of PTH1R, suggesting that these cells may be a unique phenotype that are more sensitive to PTH treatment.

Previous studies have found that intermittent or lowdose PTH (1-34) enhances bone repair and increases bone 

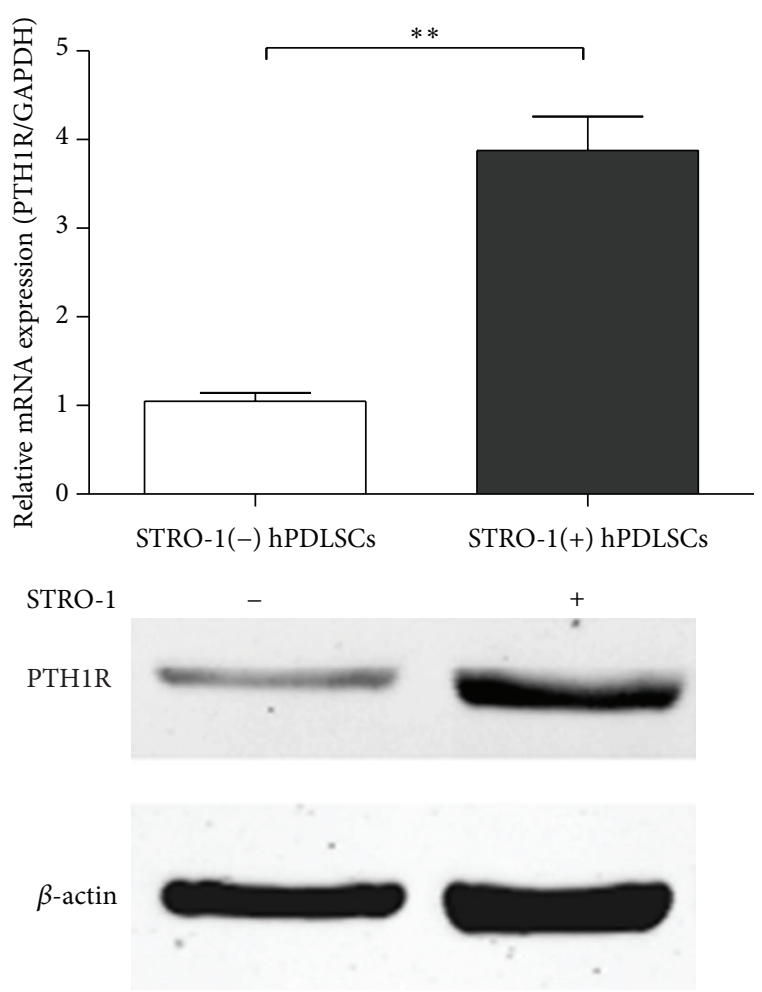

FIGURE 3: Real-time PCR and western blot analyses of PTH1R expression in STRO-1(-) and STRO-1(+) hPDLSCs. The expression of each target was normalized to that of GAPDH. Data are presented as means \pm SDs of three independent experiments performed in duplicate. ${ }^{* *} P<0.01$.

turnover [31, 32]. In our study, we used human STRO-1(+) PDLSCs as a model to explore the roles of PTH in osteogenesis. We found that PTH (1-34) increased the expression of osteoblast-related genes and the mineralization capacity of the cells, supporting the future applications of PTH in periodontitis treatment. Further studies are needed to examine the effects of PTH on periodontal tissue regeneration in vivo by transplantation of PTH-treated STRO-1(+) PDLSCs into alveolar bone defect areas in an appropriate animal model.

The functions of PTH are mediated by a G protein coupled receptor, referred to as PTH1R, the PTH-1 receptor, which regulates skeletal development, bone turnover, and mineral ion homeostasis $[33,34]$. In continuous treatment, PTH caused severe bone loss and inhibits bone related protein expression $[35,36]$, while intermittent administration of PTH enhanced bone formation [37]. These studies have shown that PTH may be a double-edged sword, suggesting that the molecular mechanisms of PTH may be highly complicated [38]. The time-dependent action of PTH might be due to differences in the signal transduction systems, indicating a synchronized regulation of cAMP/PKA and PKC signaling after PTH stimulation [39]. While the PKA pathway acts in response to short exposure to PTH, the PKC pathway is mainly involved at longer times of exposure in an antagonist mode [40, 41]. In our study, STRO-1(+) PDLSCs were treated under the intermittent administration of PTH, which might be mediated predominantly by cAMP/PKA pathway. Interestingly, our data showed that PTH upregulated its receptor PTH1R in STRO-1(+) PDLSCs, which may result in the establishment of a positive feedback loop. The observed effects could be explained by the feedback of PKA signals on PTH1R expression levels, ligand sensitivity, and crosstalk with other downstream signaling pathways [42]. However, the nature of interactions between PTH responsive signaling systems in STRO-1(+) PDLSCs remains to be further elucidated.

Runx2 is a key transcription factor involved in the process of osteogenesis and directs multipotent mesenchymal cells to the osteoblast lineage [43]. During the process of osteogenesis, $\mathrm{Sp} 7$ has proved to be a direct downstream target of RUNX2, and these two proteins physically interacted and synergistically activated osteogenic genes $[44,45]$. In our current study, PTH (1-34) treatment leads to a significant increase of RUNX2 and Sp7 after osteogenic induction. The anabolic effects of PTH on osteoblast-specific transcription factors (Runx2 and Sp7) could be attributed to the predominant activation of cAMP/PKA signal transduction [46-48]. However, our data showed that the enhancement of Sp7 expression by PTH was even more robust in both uninduced and induced STRO-1(+) cells than that of Runx2, indicating a Runx2 independent activation of Sp7 gene expression [49]. It is possible that other genes upstream of $\mathrm{Sp} 7$ may also be affected by PTH, or effects of PTH may be directly mediated through Osx and independently of Runx2 [50, 51]. Further studies are needed to fully elucidate the specific mechanisms involved in these processes.

In summary, we achieved successful culture of hPDLSCs and separation of STRO-1(+) and STRO-1(-) cells. Our additional experiments suggested that STRO-1(+) hPDLSCs had higher sensitivity to PTH and stronger osteogenic ability, which may play a major role in bone regulation in the presence of PTH. These data provide a deeper understanding of the effects of PTH on the regeneration of periodontal support tissues and may facilitate the development of improved biomaterials for periodontal stem cell treatment.

\section{Conclusion}

STRO-1(+) hPDLSCs represented a more promising cell resource for biomaterials and tissue engineering applications. Intermittent PTH treatment improved the capacity of STRO$1(+)$ hPDLSCs to repair damaged tissue and ameliorate the symptoms of periodontitis. Our results provided evidences for applications of PTH in stem cell therapy for periodontitis diseases.

\section{Competing Interests}

The authors have no competing interests to declare.

\section{Authors' Contributions}

Xiaoxiao Wang and Yanlan Wang contributed equally to this work. 

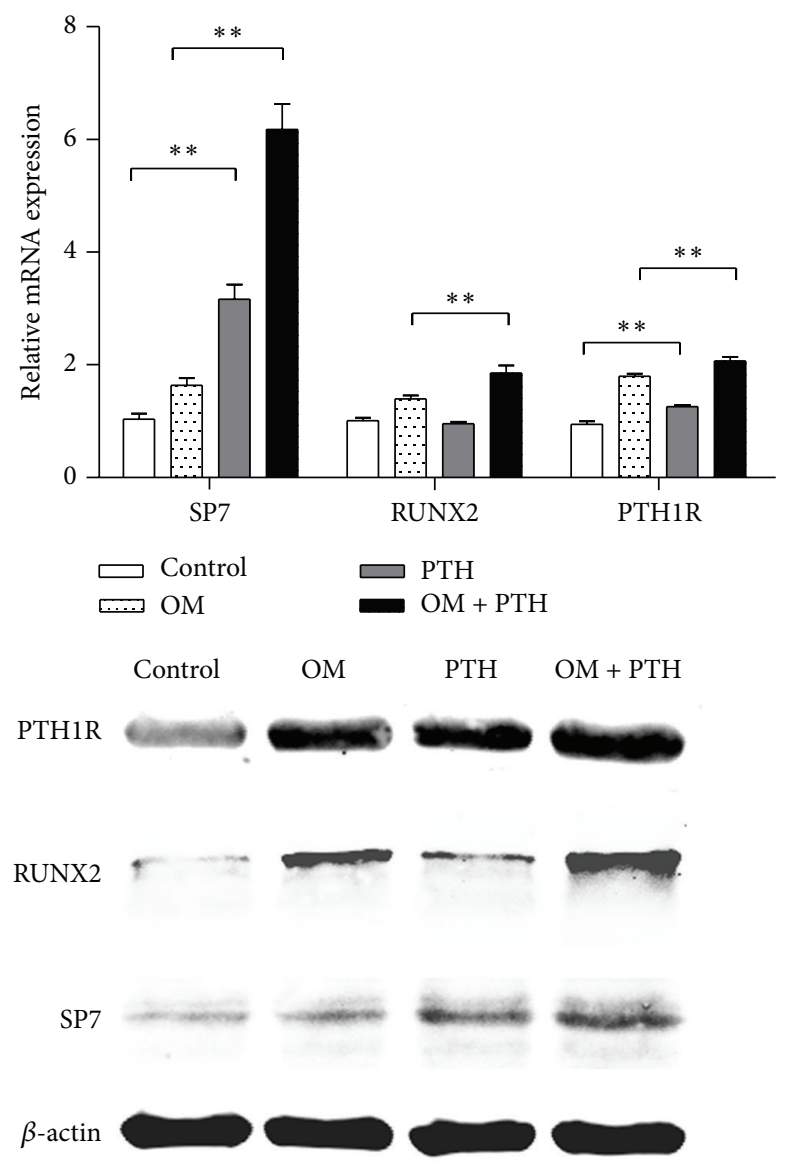

(a)
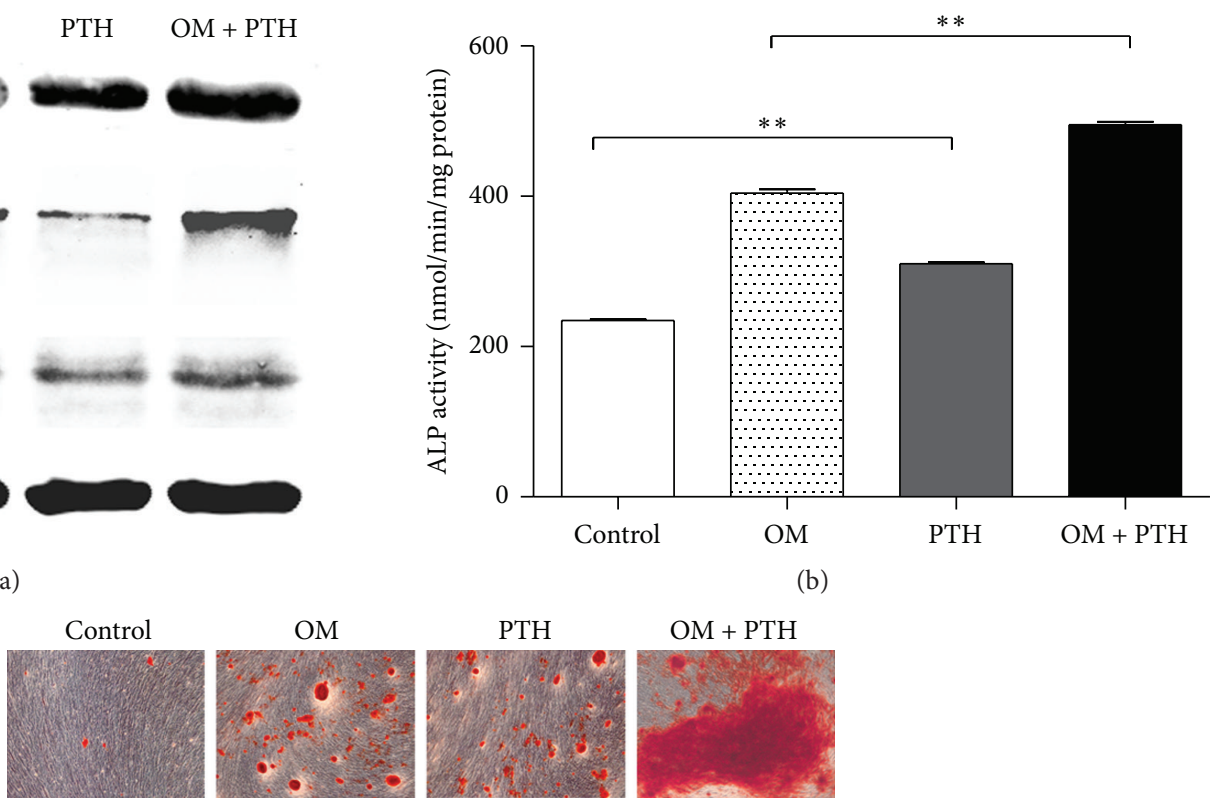

(b)

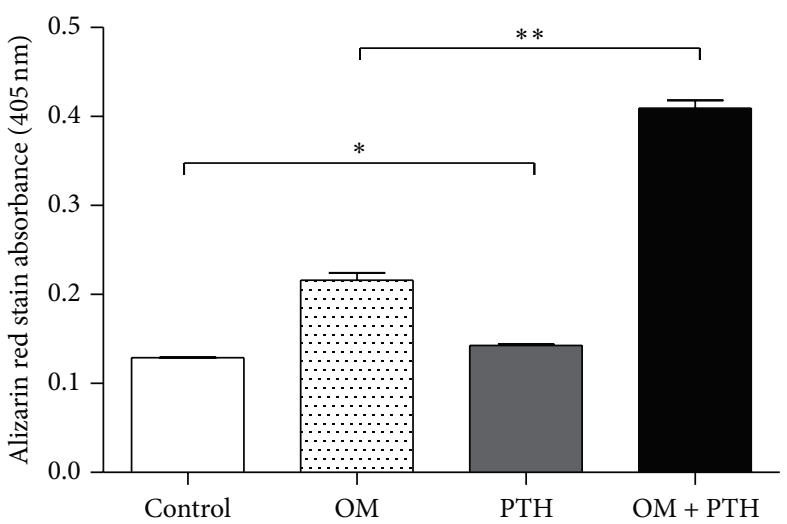

(c)

FIGURE 4: The osteogenic capacity of STRO-1(+) hPDLSCs was enhanced by PTH. (a) Real-time PCR and western blot analyses of PTH1R, RUNX2, and SP7 expression in STRO-1(+) hPDLSCs before and after PTH treatment and induction by osteogenic-induction medium (OM). The expression of each target was normalized to that of GAPDH. (b) ALP activity in STRO-1(+) hPDLSCs. (c) Cells subjected to osteogenic differentiation were stained with Alizarin red (magnification: 100x). Data are presented as the means \pm SDs of three independent experiments performed in duplicate. ${ }^{*} P<0.05,{ }^{* *} P<0.01$. 


\section{Acknowledgments}

This work was supported by the Natural Science Foundation of China (nos. 81171575 and 81371793) and the Guangdong Innovative Research Team program (no. 2009010058).

\section{References}

[1] F.-M. Chen, H.-H. Sun, H. Lu, and Q. Yu, "Stem cell-delivery therapeutics for periodontal tissue regeneration," Biomaterials, vol. 33, no. 27, pp. 6320-6344, 2012.

[2] K. Hynes, D. Menicanin, S. Gronthos, and P. M. Bartold, "Clinical utility of stem cells for periodontal regeneration," Periodontology 2000, vol. 59, no. 1, pp. 203-227, 2012.

[3] S. H. Bassir, W. Wisitrasameewong, J. Raanan et al., "Potential for stem cell-based periodontal therapy," Journal of Cellular Physiology, vol. 231, no. 1, pp. 50-61, 2016.

[4] G. Ding, Y. Liu, W. Wang et al., "Allogeneic periodontal ligament stem cell therapy for periodontitis in swine," Stem Cells, vol. 28, no. 10, pp. 1829-1838, 2010.

[5] G. Z. Racz, K. Kadar, A. Foldes et al., "Immunomodulatory and potential therapeutic role of mesenchymal stem cells in periodontitis," Journal of Physiology and Pharmacology, vol. 65, pp. 327-339, 2014.

[6] B.-M. Seo, M. Miura, S. Gronthos et al., "Investigation of multipotent postnatal stem cells from human periodontal ligament," The Lancet, vol. 364, no. 9429, pp. 149-155, 2004.

[7] J.-Y. Park, S. H. Jeon, and P.-H. Choung, "Efficacy of periodontal stem cell transplantation in the treatment of advanced periodontitis," Cell Transplantation, vol. 20, no. 2, pp. 271-285, 2011.

[8] F.-J. Lv, R. S. Tuan, K. M. C. Cheung, and V. Y. L. Leung, "Concise review: the surface markers and identity of human mesenchymal stem cells," STEM CELLS, vol. 32, no. 6, pp. 14081419, 2014.

[9] M. T. Saito, K. G. Silverio, M. Z. Casati, E. A. Sallum, and F. H. Nociti Jr., "Tooth-derived stem cells: update and perspectives," World Journal of Stem Cells, vol. 7, pp. 399-407, 2015.

[10] D. Gothard, J. Greenhough, E. Ralph, and R. O. Oreffo, "Prospective isolation of human bone marrow stromal cell subsets: a comparative study between Stro-1-, CD146- and CD105-enriched populations," Journal of Tissue Engineering, vol. 5, 2014.

[11] P. J. Psaltis, S. Paton, F. See et al., "Enrichment for STRO-1 expression enhances the cardiovascular paracrine activity of human bone marrow-derived mesenchymal cell populations," Journal of Cellular Physiology, vol. 223, no. 2, pp. 530-540, 2010.

[12] L. Wang, A. B. Tran, F. H. Nociti et al., "PTH and vitamin D repress DMP1 in cementoblasts," Journal of Dental Research, vol. 94, no. 10, pp. 1408-1416, 2015.

[13] B. Yu, X. Zhao, C. Yang et al., "Parathyroid hormone induces differentiation of mesenchymal stromal/stem cells by enhancing bone morphogenetic protein signaling," Journal of Bone and Mineral Research, vol. 27, no. 9, pp. 2001-2014, 2012.

[14] M. I. Almagro, J. A. Roman-Blas, M. Bellido, S. Castañeda, R. Cortez, and G. Herrero-Beaumont, "PTH [1-34] enhances bone response around titanium implants in a rabbit model of osteoporosis," Clinical Oral Implants Research, vol. 24, no. 9, pp. 1027-1034, 2013.

[15] D. F. P. Vasconcelos, M. R. Marques, B. B. Benatti, S. P. Barros, F. H. Nociti Jr., and P. D. Novaes, "Intermittent parathyroid hormone administration improves periodontal healing in rats," Journal of Periodontology, vol. 85, no. 5, pp. 721-728, 2014.
[16] C. A. Gregory, W. G. Gunn, A. Peister, and D. J. Prockop, “An Alizarin red-based assay of mineralization by adherent cells in culture: comparison with cetylpyridinium chloride extraction," Analytical Biochemistry, vol. 329, no. 1, pp. 77-84, 2004.

[17] M. Sakaki-Yumoto, J. Liu, M. Ramalho-Santos, N. Yoshida, and R. Derynck, "Smad2 is essential for maintenance of the human and mouse primed pluripotent stem cell state," The Journal of Biological Chemistry, vol. 288, no. 25, pp. 18546-18560, 2013.

[18] R.-P. Zhang, J.-Z. Shao, and L.-X. Xiang, “GADD45A protein plays an essential role in active DNA demethylation during terminal osteogenic differentiation of adipose-derived mesenchymal stem cells," The Journal of Biological Chemistry, vol. 286, no. 47, pp. 41083-41094, 2011.

[19] S. Nozell and X. Chen, "P21B, a variant of p21(Waf1/Cip1), is induced by the p53 family," Oncogene, vol. 21, no. 8, pp. 1285$1294,2002$.

[20] M. Dominici, K. Le Blanc, I. Mueller et al., "Minimal criteria for defining multipotent mesenchymal stromal cells. The International Society for Cellular Therapy position statement," Cytotherapy, vol. 8, no. 4, pp. 315-317, 2006.

[21] J.-C. Park, J.-M. Kim, I.-H. Jung et al., "Isolation and characterization of human periodontal ligament (PDL) stem cells (PDLSCs) from the inflamed PDL tissue: in vitro and in vivo evaluations," Journal of Clinical Periodontology, vol. 38, no. 8, pp. 721-731, 2011.

[22] A. Nasef, Y. Z. Zhang, C. Mazurier et al., "Selected Stro-1enriched bone marrow stromal cells display a major suppressive effect on lymphocyte proliferation," International Journal of Laboratory Hematology, vol. 31, no. 1, pp. 9-19, 2009.

[23] Y. Zhang, D. Zhao, C. Tian et al., "Stro-1-positive human mesenchymal stem cells prolong skin graft survival in mice," Transplantation Proceedings, vol. 45, no. 2, pp. 726-729, 2013.

[24] M. Lei, K. Li, B. Li, L.-N. Gao, F.-M. Chen, and Y. Jin, "Mesenchymal stem cell characteristics of dental pulp and periodontal ligament stem cells after in vivo transplantation," Biomaterials, vol. 35, no. 24, pp. 6332-6343, 2014.

[25] Q. Xu, B. Li, L. Yuan et al., "Combination of platelet-rich plasma within periodontal ligament stem cell sheets enhances cell differentiation and matrix production," Journal of Tissue Engineering and Regenerative Medicine, 2014.

[26] L. Du, P. Yang, and S. Ge, "Stromal cell-derived factor1 significantly induces proliferation, migration, and collagen type $\mathrm{i}$ expression in a human periodontal ligament stem cell subpopulation," Journal of Periodontology, vol. 83, no. 3, pp. 379-388, 2012.

[27] K. M. El-Sayed, S. Paris, C. Graetz et al., "Isolation and characterisation of human gingival margin-derived STRO-1/MACS ${ }^{+}$ and MACS $^{-}$cell populations," International Journal of Oral Science, vol. 7, no. 2, pp. 80-88, 2014.

[28] F. Kantawong, K. E. V. Burgess, K. Jayawardena et al., "Effects of a surface topography composite with puerariae radix on human STRO-1-positive stem cells," Acta Biomaterialia, vol. 6, no. 9, pp. 3694-3703, 2010.

[29] L. Du, P. Yang, and S. Ge, "Stromal cell-derived factor1 significantly induces proliferation, migration, and collagen type I expression in a human periodontal ligament stem cell subpopulation," Journal of Periodontology, vol. 83, no. 3, pp. 379-388, 2012.

[30] J. Xu, W. Wang, Y. Kapila, J. Lotz, and S. Kapila, "Multiple differentiation capacity of STRO- $1^{+} / \mathrm{CD} 146^{+}$PDL mesenchymal progenitor cells," Stem Cells and Development, vol. 18, no. 3, pp. 487-496, 2009. 
[31] O. Sandberg, B. R. Macias, and P. Aspenberg, "Low dose PTH improves metaphyseal bone healing more when muscles are paralyzed," Bone, vol. 63, pp. 15-19, 2014.

[32] M. Takahata, H. A. Awad, R. J. O’Keefe, S. V. Bukata, and E. M. Schwarz, "Endogenous tissue engineering: PTH therapy for skeletal repair," Cell and Tissue Research, vol. 347, no. 3, pp. 545552, 2012.

[33] R. L. Jilka, "Molecular and cellular mechanisms of the anabolic effect of intermittent PTH," Bone, vol. 40, no. 6, pp. 1434-1446, 2007.

[34] R. W. Cheloha, S. H. Gellman, J. P. Vilardaga, and T. J. Gardella, "PTH receptor-1 signalling mechanistic insights and therapeutic prospects," Nature Reviews Endocrinology, vol. 11, no. 12, pp. 712-724, 2015.

[35] Y. L. Ma, R. L. Cain, D. L. Halladay et al., "Catabolic effects of continuous human PTH (1-38) in vivo is associated with sustained stimulation of RANKL and inhibition of osteoprotegerin and gene-associated bone formation," Endocrinology, vol. 142, no. 9, pp. 4047-4054, 2001.

[36] S. H. H. Hong, X. Lu, M. S. Nanes, and J. Mitchell, "Regulation of osterix (Osx, Sp7) and the Osx promoter by parathyroid hormone in osteoblasts," Journal of Molecular Endocrinology, vol. 43, no. 5, pp. 197-207, 2009.

[37] Q. Liu, Q. Wan, R. Yang, H. Zhou, and Z. Li, "Effects of intermittent versus continuous parathyroid hormone administration on condylar chondrocyte proliferation and differentiation," Biochemical and Biophysical Research Communications, vol. 424, no. 1, pp. 182-188, 2012.

[38] L. Qin, L. J. Raggatt, and N. C. Partridge, "Parathyroid hormone: a double-edged sword for bone metabolism," Trends in Endocrinology and Metabolism, vol. 15, no. 2, pp. 60-65, 2004.

[39] T. Ishizuya, S. Yokose, M. Hori et al., "Parathyroid hormone exerts disparate effects on osteoblast differentiation depending on exposure time in rat osteoblastic cells," The Journal of Clinical Investigation, vol. 99, no. 12, pp. 2961-2970, 1997.

[40] G. N. Guimarães, T. L. Rodrigues, A. P. De Souza, S. R. Line, and M. R. Marques, "Parathyroid hormone (1-34) modulates odontoblast proliferation and apoptosis via PKA and PKCdependent pathways," Calcified Tissue International, vol. 95, no. 3, pp. 275-281, 2014.

[41] N. Abuduwali, S. Lossdörfer, J. Winter et al., "Functional characterization of the parathyroid hormone 1 receptor in human periodontal ligament cells," Clinical Oral Investigations, vol. 18, no. 2, pp. 461-470, 2014.

[42] J. Fischer, A. Aulmann, V. Dexheimer, T. Grossner, and W. Richter, "Intermittent PTHrP(1-34) exposure augments chondrogenesis and reduces hypertrophy of mesenchymal stromal cells," Stem Cells and Development, vol. 23, no. 20, pp. 2513-2523, 2014.

[43] T. Komori, "Regulation of bone development and extracellular matrix protein genes by RUNX2," Cell and Tissue Research, vol. 339, no. 1, pp. 189-195, 2010.

[44] H. Rashid, C. Ma, H. Chen et al., "Sp7 and Runx2 molecular complex synergistically regulate expression of target genes," Connective Tissue Research, vol. 55, supplement 1, pp. 83-87, 2014.

[45] J.-E. Baek, J.-Y. Choi, and J.-E. Kim, "Skeletal analysis and differential gene expression in Runx2/Osterix double heterozygous embryos," Biochemical and Biophysical Research Communications, vol. 451, no. 3, pp. 442-448, 2014.

[46] B. L. Wang, C. L. Dai, J. X. Quan et al., "Parathyroid hormone regulates osterix and Runx2 mRNA expression predominantly through protein kinase A signaling in osteoblast-like cells," Journal of Endocrinological Investigation, vol. 29, no. 2, pp. 101108, 2006.

[47] V. Krishnan, T. L. Moore, Y. L. Ma et al., "Parathyroid hormone bone anabolic action requires cbfal/runx2-dependent signaling," Molecular Endocrinology, vol. 17, no. 3, pp. 423-435, 2003.

[48] S. Vimalraj, B. Arumugam, P. Miranda, and N. Selvamurugan, "Runx2: structure, function, and phosphorylation in osteoblast differentiation," International Journal of Biological Macromolecules, vol. 78, pp. 202-208, 2015.

[49] Q. Tu, P. Valverde, and J. Chen, "Osterix enhances proliferation and osteogenic potential of bone marrow stromal cells," Biochemical and Biophysical Research Communications, vol. 341, no. 4, pp. 1257-1265, 2006.

[50] L. A. Kaback, D. Y. Soung, A. Naik et al., "Teriparatide (134 human PTH) regulation of osterix during fracture repair," Journal of Cellular Biochemistry, vol. 105, no. 1, pp. 219-226, 2008.

[51] R. Barbuto and J. Mitchell, "Regulation of the osterix (Osx, Sp7) promoter by osterix and its inhibition by parathyroid hormone," Journal of Molecular Endocrinology, vol. 51, no. 1, pp. 99-108, 2013. 

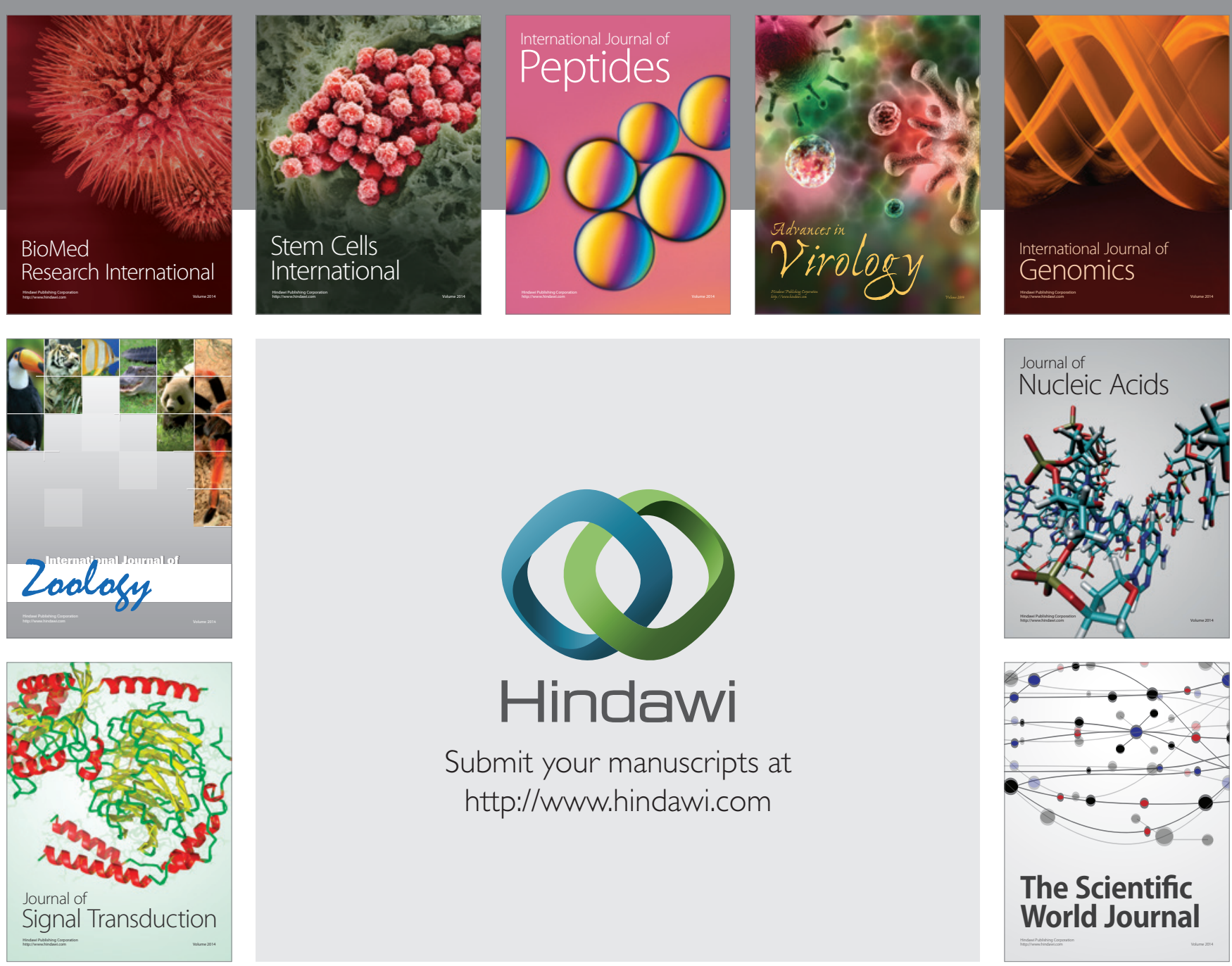

Submit your manuscripts at

http://www.hindawi.com
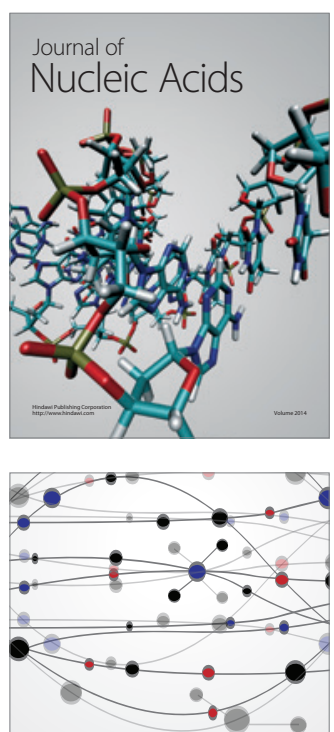

The Scientific World Journal
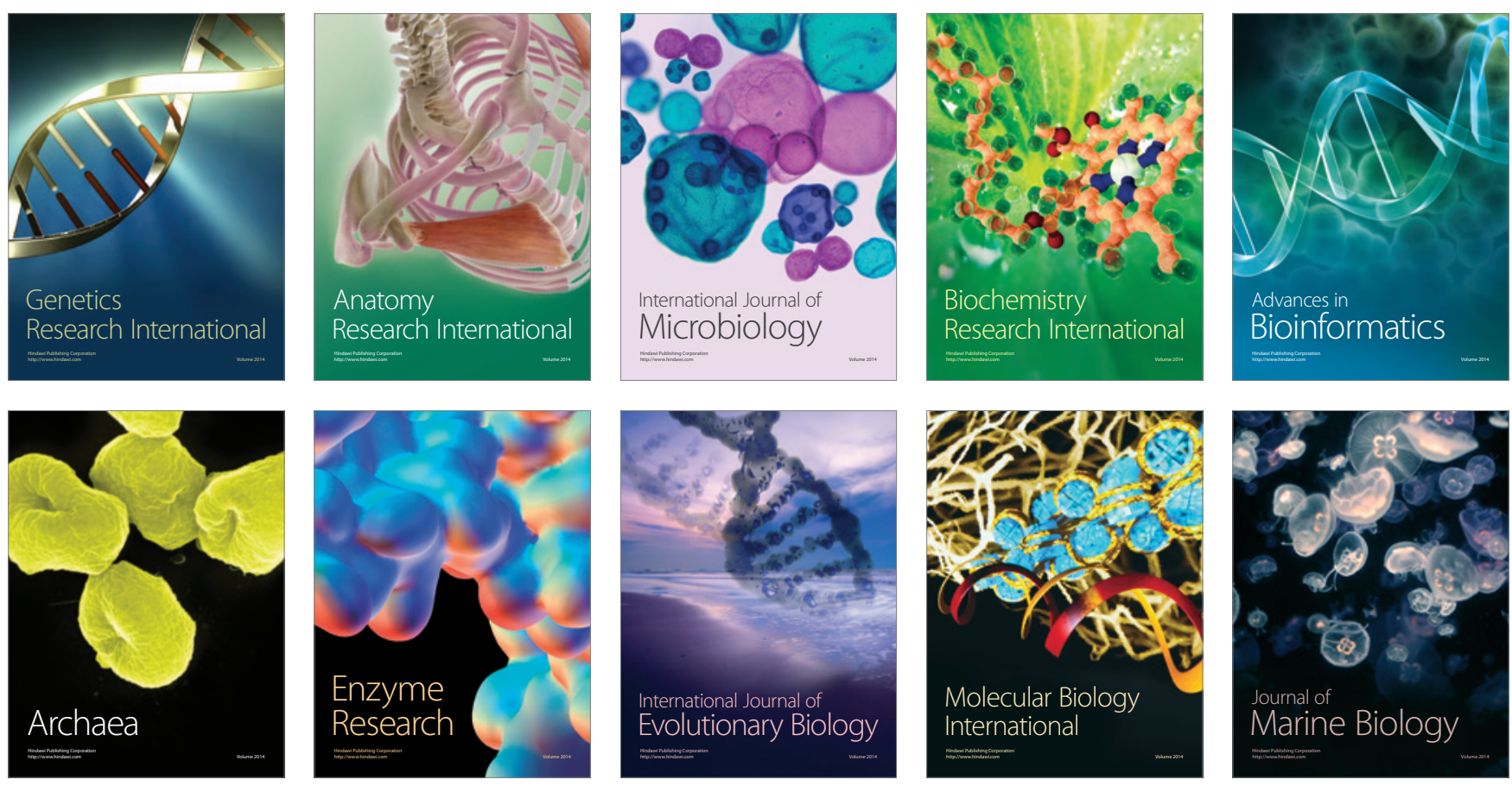